\title{
Particle-Based Approach for Simulation of Nonlinear Material Behavior in Contact Zones
}

\author{
Evgeny V. Shilko, Alexey Yu. Smolin, Andrey V. Dimaki, \\ and Galina M. Eremina
}

\begin{abstract}
Methods of particles are now recognized as an effective tool for numerical modeling of dynamic mechanical and coupled processes in solids and liquids. This chapter is devoted to a brief review of recent advances in the development of the popular particle-based discrete element method (DEM). DEM is conventionally considered as a highly specialized technique for modeling the flow of granular media and the fracture of brittle materials at micro- and mesoscopic scales. However, in the last decade, great progress has been made in the development of the formalism of this method. It is largely associated with the works of the scientific group of Professor S. G. Psakhie. The most important achievement of this group is a generalized formulation of the method of homogeneously deformable discrete elements. In the chapter, we describe keystones of this implementation of DEM and a universal approach that allows one to apply various rheological models of materials (including coupled models of porous fluid-saturated solids) to a discrete element. The new formalism makes possible qualitative expansion of the scope of application of the particle-based discrete element technique to materials with various rheological properties and to the range of considered scales form microscopic to macroscopic. The capabilities of this method are especially in demand in the study of the features of contact interaction of materials. To demonstrate these capabilities, we briefly review two recent applications concerning (a) the effect of adhesive interaction on the regime of wear of surface asperities under tangential contact of bodies and (b) the nonmonotonic dependence of the stress concentration in the neck of the human femur on the dynamics of hip joint contact loading.
\end{abstract}

Keywords Discrete element method - Deformable element $\cdot$ Movable cellular automata $\cdot$ Many-body interaction $\cdot$ Plasticity $\cdot$ Poroelasticity $\cdot$ Surface adhesion $\cdot$ Adhesive wear $\cdot$ Bone tissue $\cdot$ Pore fluid

\footnotetext{
E. V. Shilko (凶) - A. Yu. Smolin · A. V. Dimaki · G. M. Eremina

Institute of Strength Physics and Materials Science SB RAS, 634055 Tomsk, Russia

e-mail: shilko@ispms.tsc.ru
} 


\section{Introduction}

Starting with the classic works of Cauchy and Navier [1, 2], the development of the formalism of the discrete representation of the medium at a continuum ("superatomic") scale is considered among the fundamental problems for the mechanics of solids. In the framework of this representation, a matter is described by an ensemble of interacting particles. Each particle models a sufficient number of atoms or molecules to describe the state and response of the particle in terms of thermodynamic parameters and classical mechanical models (Fig. 1). A discrete description of solids and liquids was initially considered as a way to fill the gap between molecular mechanics and continuum mechanics [3]. However, the rapid development of the formalism of particle methods in the last two decades has made it possible to apply them to study the mechanical behavior of diverse solids as well as various mechanically assisted or activated processes in the entire spectrum of spatial scales from atomic to macroscopic.

The traditional approach to the numerical study of the behavior of materials on the "above-atomic" spatial scales is based on the methods of continuum mechanics such as finite element, finite difference and boundary element methods (FEM, FDM and BEM) [4-9]. The formalism of these methods allows easy implementation of various linear and nonlinear (including coupled thermomechanical and poroelastic) rheological models. Moreover, advanced implementations of FEM, FDM and BEM include the ability to directly model fracture $[10,11]$. Despite the well-known advantages of continuum numerical methods, their fundamental limitation is difficulty in modeling of complex fracture-related problems including development of multiple fractures, contact interaction of the initial and newly formed surfaces, wear of surface layers and a change in surface roughness, flow of granular media, etc.

Mentioned limitation is not inherent in particle-based methods [12-19]. The most relevant and efficient particle-based method for numerical modeling of the abovesaid complex mechanical processes in solids is the discrete element method (DEM) $[18,19]$. The constantly growing interest in this numerical technique is determined by the ability to solve a variety of complex and non-linear contact problems, where the processes of fragmentation and mass transfer of fragments play a key role. This

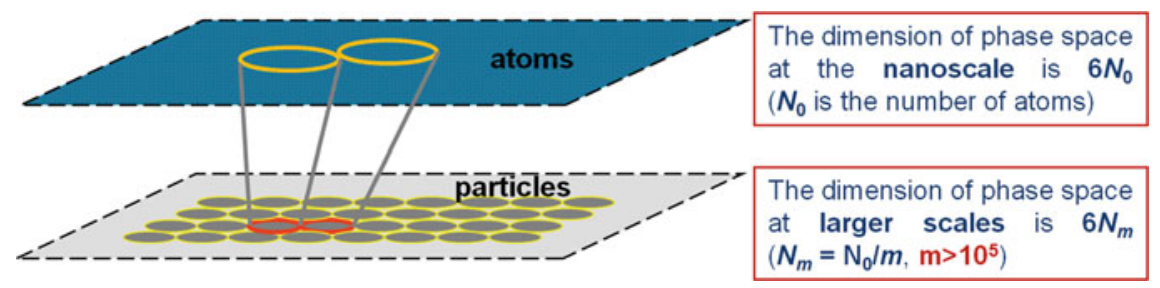

Fig. 1 Representation of the material by ensemble of particles as an extension of atomistic representation to higher spatial (and temporal) scales 
chapter is devoted to the analysis of achievements in the development of DEM for computer simulation of the mechanical behavior of consolidated solids.

Creation of this method is attributed to Cundall [20, 21]. In the framework of original implementation of DEM, a discrete element is treated as a finite part of a solid body (or a particle in particulate/granular material) bounded by clearly defined (exact) surface. The latter qualitatively distinguishes this method from the methods of quasiparticles with "fuzzy" surface. Elements can be either chemically bonded if they model a consolidated material, or contact if the contact interaction of the fragments is modeled. Changing the type of bond of elements (chemically bonded $\leftrightarrow$ contact $\leftrightarrow$ noninteracting) is governed by the applied criteria of fracture, bond formation and contact loss $[22,23]$. The stress state of a discrete element is determined by the mechanical load of the surrounding elements on the surface of the element. The absence of a constraint in the form of the continuity equation makes the DEM extremely attractive for numerical studying complex processes in contact zones.

The term "discrete element method" is now used as a generic name for a large group of numerical techniques based on these general principles for representing the medium [18, 21, 24, 25]. Various representatives of this group differ in several key features: the principle of local or global force equilibrium (explicit or implicit formulation); approximation of the shape of the volume modeled by a discrete element; approximation to the description of the deformability of a discrete element.

Explicit DEM is the most popular and is widely used to solve fracture- and contact interaction related dynamic problems. It implies the formulation of equations of motion for each discrete element and the parallel solution of the system of these equations with an explicit time marching scheme (Euler, Verlet or other integration algorithm). Since a discrete element simulates a finite volume of material, the mechanical interaction of such finite volumes should lead not only to their translational motion, but also to rotation determined by the moments of interaction forces. The form of the dynamics equations for the rotational degrees of freedom of an element (Euler's equations) is determined by the shape of an element. In the general case, Euler's equations are written in integral form using the inertia tensor (tensor of inertia, in turn, is formulated as an integral) [18]. Integral Euler equation is cumbersome and computationally costly, that is why elements with complex nonequiaxial geometry (polygonal [26], superquadric [27] elements) are used only for modeling granular or fragmented (block-structured) materials. At the same time, a consolidated material can be much more efficiently modelled by an ensemble of bonded equiaxial elements, the shape of which is approximated by equivalent sphere $(3 \mathrm{D}$ problem) or a disk of a given height (2D problem) $[18,22,28]$. The efficiency of an approximation of an equivalent disk/sphere is determined by the simplicity of the Newton-Euler motion equations for a discrete element:

$$
\left\{\begin{array}{l}
m_{i} \frac{d^{2} \vec{r}_{i}}{d t^{2}}=m_{i} \frac{d \vec{v}_{i}}{d t}=\vec{F}_{i}=\sum_{k=1}^{N_{i}} \vec{F}_{i k}=\sum_{k=1}^{N_{i}}\left(\vec{F}_{i k}^{c}+\vec{F}_{i k}^{t}\right) \\
J_{i} \frac{d \vec{\omega}_{i}}{d t}=\vec{M}_{i}=\sum_{k=1}^{N_{i}} \vec{M}_{i k}
\end{array},\right.
$$


where $i$ is the number of discrete element, $\vec{r}_{i}, \vec{v}_{i}$ and $\vec{\omega}_{i}$ are the radius-vector, velocity vector and angular velocity pseudovector respectively, $m_{i}$ is the mass of the element $i, J_{i}$ is moment of inertia of an equivalent disk or sphere, $\vec{F}_{i}$ and $\vec{M}_{i}$ are the total force and torque acting on the element $i$ by the neighbors, $\vec{F}_{i k}$ is the force of interaction of the considered element $i$ with the neighbor $k, \vec{F}_{i k}^{c}$ and $\vec{F}_{i k}^{t}$ are the central (along the line connecting mass centers of the elements $i$ and $k$ ) and tangential (in transverse plane) components of the force $\vec{F}_{i k}, \vec{M}_{i k}$ is the moment of interaction forces (includes tangential moment of $\vec{F}_{i k}^{t}$ and twisting moment [22]), $N_{i}$ is the number of neighbors of the element $i$.

One can see that the approximation of the equivalent disk/sphere allows the use of Euler's equations in the most trivial and computationally efficient form. Another important consequence of this approximation is the formal independence of the central and tangential interactions: the force $\vec{F}_{i k}^{c}$ does not cause acceleration in the plane of the tangential interaction, and the force $\vec{F}_{i k}^{t}$ does not cause acceleration along the line connecting the centers of mass of the elements.

The forces $\vec{F}_{i k}^{c}$ and $\vec{F}_{i k}^{t}$ of interaction of discrete elements are traditionally represented as the sum of the potential $\left(\vec{F}_{i k}^{c p}\right.$ and $\left.\vec{F}_{i k}^{t p}\right)$ and viscous $\left(\vec{F}_{i k}^{c v}\right.$ and $\left.\vec{F}_{i k}^{t v}\right)$ constituents [20, 22]. From the point of view of the rheological description, viscous forces have a meaning similar to the physical meaning of the damper in the KelvinVoight viscoelastic model. A key component of constructing a discrete-element model of a material is the determination of the structural type and coefficients of the potential interaction forces.

In the framework of the traditional DEM implementation, the central and tangential potential forces of interaction of equivalent balls/disks $\left(F_{i k}^{c p}\right.$ and $\left.F_{i k}^{t p}\right)$ are calculated in the pair-wise approximation. From the physical point of view, pair-wise potential corresponds to the approximation of a non-deformable (rigid) element (a system of springs or rods). Here, the term "rigid" is used in the sense that the interaction of the element $i$ with the neighbor $k$ does not change its volume and shape and, therefore, does not cause a change in the forces of interaction of the element $i$ with other neighbors (these neighbors "feel" the result of the interaction in the pair $i-k$ only indirectly, through the motion of the element $i$ ). This approximation is widely used for micro- and mesoscopic description of the processes of damage accumulation and fracture of brittle materials, contact interaction of elastic bodies, including the dynamics of block-structured media. In this case, element-element interaction is traditionally modelled using harmonic interaction potentials (such an interaction is schematically represented by connecting the centers of the elements with two springs oriented in the central and tangential directions) [22, 28, 29]. Some models also use nonlinear (elastic-plastic or viscoelastic Maxwell type) formulations of pair-wise interaction forces $F_{i k}^{c p}$ and $F_{i k}^{t p}[30,31]$ for simulation of granular media and porous structures with non-linear/ductile rheological properties of the material of the skeleton walls or granules. However, such potentials make possible adequate description of the mechanical behavior of porous systems only at a "low" scale (the scale of discontinuities or granules). 
The key problems that strongly limit the range of application of the traditional implementation of DEM with pair-wise interaction forces are well known. They are (i) dependence of the macroscopic properties of an ensemble of discrete elements on the type of packaging and size distribution of elements, (ii) incorrect description of the plastic strain of an ensemble of elements (for example, plastic deformation of a sample may be accompanied by an uncontrolled change in its volume), and other related problems.

Various approaches to solving these fundamental difficulties within the framework of the concept of non-deformable elements have been proposed in last decades. In particular, stochastic dense packing of non-uniform-sized circular (2D) or spherical (3D) elements [22, 28, 29] is used to solve the problems of packing-induced anisotropy of the elastic response and packing-dependent ratio of elastic modules of an ensemble of elements. An alternative approach is to use the formalism of spring network model (lattice model $[32,33]$ ) to build relationships for the forces of interaction of regularly packed uniform-sized elements [34-36]. The lattice model is based on the postulation of the form of interaction potential (harmonic potential is usually used for both central and angular interactions) and equalization of elastic strain energy stored in a unit cell of volume to the associated elastic strain energy of the modelled continuum. The material parameters derived from this equality are included in the relationships for the forces of element-element interaction. The above approaches made it possible to adequately describe the mechanical (and thermomechanical [36]) behavior of brittle materials under complex loading conditions. At the same time, they do not allow solving the key problem of incorrect modeling of nonlinear (and/or inelastic) mechanical behavior of materials with complex rheological properties (including rubber-like viscoelastic materials as well as metallic and polymer materials, whose macroscopic plasticity is not related to discontinuities).

The problem of correct modeling of nonlinear mechanical behavior of consolidated materials by the method of discrete elements can be generally solved only by using the approximation of the deformable element. In turn, the deformability of an element can be realized only within the framework of a many-body interaction of elements. This means that the potential interaction force must depend not only on the relative motion of the elements in the pair, but also on the interaction of each of them with other neighbors. The formulation of the general structural form of the potential interaction force and its specific realizations for materials with various rheological properties has been among the critical challenges for the DEM until recently.

\section{Distinct Element Method with Deformable Elements}

A meaningful contribution to the development of the formalism of DEM was done by Professor Sergey G. Psakhie and his team. Professor Psakhie was a founder of the new particle-based method, namely, the method of movable cellular automata (MCA) [37, 38].The basic principles of this method were developed in collaboration with Professor Yuki Horie (North Carolina State University, Los Alamos National 
Lab). Originally, the MCA method was designed as a hybrid technique to model mechanically activated chemical reactions in powder mixtures $[39,40]$. This original implementation combined the formalisms of discrete elements and cellular automata, in which the mechanical response of the particle was described using the DEM formalism, while the non-mechanical thermodynamic aspects of particle-particle interaction (including melting and mechanically activated chemical reaction) were modelled on the basis of the concept of cellular automata.

The most important achievement of S. G. Psakhie in the development of numerical particle-based modelling techniques is the proposed general formalism of the method of homogeneously (simply) deformable discrete elements.

The keystones of this formalism were laid in the framework of collaboration with Professor Valentin L. Popov (Technische Universität Berlin) and his scientific group. In a joint work of Professors Psakhie and Popov [41], the basic principles for describing the mechanical behavior of a discrete element (movable cellular automaton) as a deformable area of the medium were formulated. For a special case of an ensemble of close packed elements of the same equivalent radius, which models an isotropic two-dimensional continuum, a relation was proposed for the potential force of the central interaction of elements in the many-body approximation:

$$
F_{i k}^{c p}=E^{*} \delta L_{i k}=E^{*}\left(\delta r_{i k}+D \sum_{j=1}^{N} \delta r_{i j}+D \sum_{m=1}^{N} \delta r_{k m}\right) \text {. }
$$

Here, the symbol $\delta$ denotes the difference between the current and initial values of the corresponding parameter, $r_{i k}=\left|\vec{r}_{i}-\vec{r}_{k}\right|$ is the distance between the centers of mass of the elements $i$ and $k, L_{i k}$ is the effective distance between the elements, $N$ is the number of neighbors in the first coordination sphere. The coefficients $E^{*}$ and $D$ are expressed in terms of the elastic constants of the material and the element packing parameters. Derivation of these coefficients is based on the condition for ensuring the required values of Young's modulus and Poisson's ratio of the material [41]. This model is based on the same principles as the classic spring network models, but it has a fundamental difference. The force of the central interaction of the two elements is represented in the form of a superposition of the pair-wise component $E^{*} \delta r_{i k}$ and "hydrostatic" components. The latter are proportional to the change in the volumes of the interacting elements (here, we use the particular form of expression for the element's volume change in regular packing). The reasonableness of this formulation is confirmed by the linear relationship of the diagonal components of the stress tensor and the volume strain in the vast majority of macroscopic rheological models of materials (linear and non-linear elasticity, viscoelasticity, plasticity).

The proposed formalism actually uses the approximation of homogeneously deformable elements. It was further developed to describe plastic flow of the elements based on constitutive equations of the macroscopic continuum theory of defects [41]. Despite the clear advantages of the proposed formalism, it has the same key limitations as traditional lattice-based models. Among them, are the absence of the 
tangential interaction of elements (shear resistance force), packing-dependent artificial anisotropy of the integral response of the ensemble of elements at a significant distortion of the initial symmetry of the lattice, and the lack of a general and simple algorithm of implementation of complex rheological material models.

A generalized formulation of the method of homogeneously deformable discrete elements was proposed later in the works of Professor S. G. Psakhie with co-authors. It applies the concept of many-body interaction for the ensemble of arbitrarily packed different-size elements and is based on the following principles:

1. Approximation of equivalent disks/spheres (Fig. 2). Within the framework of this approximation, the dynamics of elements is described by Eq. (1), and the forces of central and tangential interaction are assumed to be formally unrelated to each other. Elements interact with each other through flat contact areas. The geometry and squares of these areas are determined by the local packing and sizes of the elements $[22,42,43]$. The potential interaction of the two elements is conveniently described in terms of specific forces of interaction (normal $\sigma_{i k}$ and tangential $\tau_{i k}$ contact stresses):

$$
\left\{\begin{array}{l}
F_{i k}^{c p}=\sigma_{i k} S_{i k} \\
F_{i k}^{t p}=\tau_{i k} S_{i k}
\end{array} .\right.
$$

2. A discrete element is assumed to be homogeneously deformable, i.e., its stressstrain state is characterized by tensors of stress and strain (hereinafter called

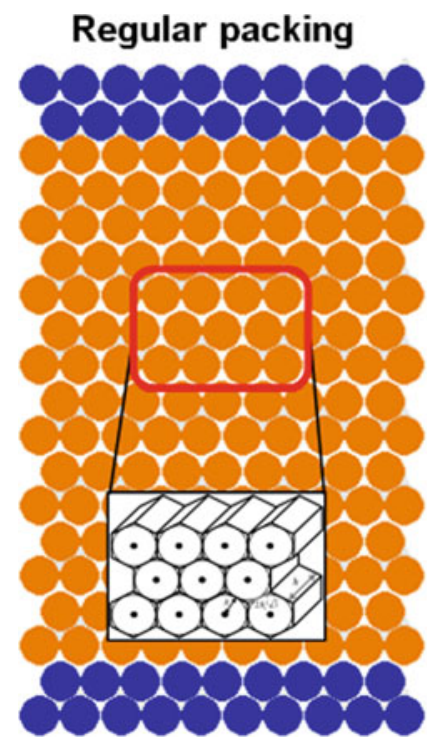

\section{Stochastic packing}

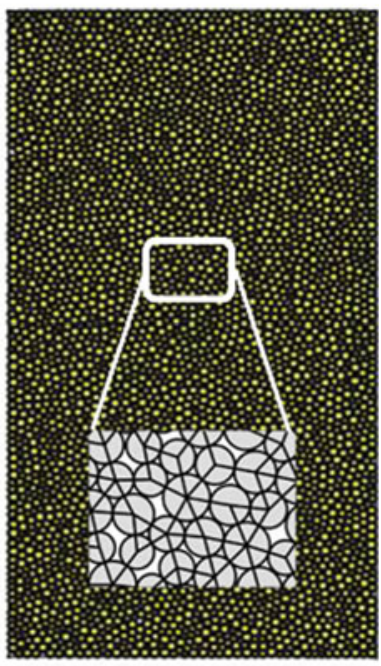

Fig. 2 Typical examples of 2D samples modelled by the ensembles of regularly and stochastically (dense) packed discrete elements. Figures show equivalent disks 
average stresses $\bar{\sigma}_{\alpha \beta}$ and strains $\bar{\varepsilon}_{\alpha \beta}$, where $\alpha, \beta=x, y, z$ ). To determine the components of average stress tensor, we use well-known classical relations for average stresses and "local" values of traction vectors at the contact areas of the element with neighbors (stress homogenization) [22, 42, 44]:

$$
\bar{\sigma}_{\alpha \beta}^{i}=\frac{R_{i}}{\Omega_{i}^{0}} \sum_{k=1}^{N_{i}} S_{i k}^{0}\left(\vec{n}_{i k}\right)_{\alpha}\left(\vec{\Sigma}_{i k}\right)_{\beta}=\frac{R_{i}}{\Omega_{i}^{0}} \sum_{k=1}^{N_{i}} S_{i k}^{0}\left(\vec{n}_{i k}\right)_{\alpha}\left(\sigma_{i k}\left(\vec{n}_{i k}\right)_{\beta}+\tau_{i k}\left(\vec{t}_{i k}\right)_{\beta}\right) \text {, }
$$

where $\mathrm{R}_{i}$ is the radius of equivalent sphere approximating the element $i, \Omega_{i}^{0}$ is the volume of unstrained element, $S_{i k}^{0}$ is the contact square in unstrained pair $i-k, \vec{\Sigma}_{i k}$ is the traction vector at the area of contact of elements $i$ and $k$ (normal and tangential contact stresses are the components of this vector), $\vec{n}_{i k}$ is the unit normal vector directed along the line connecting the mass centers of the elements, $\vec{t}_{i k}$ is the unit tangent vector directed in the tangential plane, $(\vec{W})_{\beta}$ is the projection of some vector $\vec{W}$ onto the $\beta$-axis. We emphasize the generality of definition (4), which is applicable for arbitrary local packing of elements of various sizes (packing and sizes determine the values of the direction cosines and contact areas).

3. A consequence of the deformability of an element is the need to divide the spatial parameters of its interaction with a neighbor (pair overlap and relative tangential displacement) into two components, namely, the contributions of both elements:

$$
\left\{\begin{array}{l}
\Delta r_{i k}=\Delta q_{i k}+\Delta q_{k i}=R_{i} \Delta \varepsilon_{i k}+R_{k} \Delta \varepsilon_{k i} \\
\Delta l_{i k}^{s h}=R_{i} \Delta \gamma_{i k}+R_{k} \Delta \gamma_{k i}
\end{array}\right.
$$

where $q_{i k}$ and $q_{k i}$ are the distances from the mass centers of the interacting elements $i$ and $k$ to the central point of the contact area (they are equal to equivalent radii $R_{i}$ and $R_{k}$ respectively for the case of unstrained elements), $\varepsilon_{i k}$ and $\varepsilon_{k i}$ are central pair strains of discrete elements $i$ and $k, l_{i k}^{\text {shear }}$ is the value of relative tangential displacement of the elements (it is calculated with taking into account the element rotations $[22,39,42]), \gamma_{i k}$ and $\gamma_{k i}$ are the shear angles of discrete elements $i$ and $k$ (contributions to the total shear angle). In the general case, $\varepsilon_{i k} \neq \varepsilon_{k i}$ and $\gamma_{i k} \neq \gamma_{k i}$. Relations (5) are given in the incremental form [hereinafter the symbol $\Delta$ denotes an increment of some parameter over the time step of numerical integration of the motion equations (1)] because this form is convenient for the numerical implementation of complex rheological models. Note that the strains $\varepsilon_{i k}$ and $\gamma_{i k}$ are the components of the "local" strain vector, which is used in the definition of $\bar{\varepsilon}_{\alpha \beta}^{i}$ by the analogy to (4).

4. In the framework of approximation of deformable element, the specific normal and tangential forces (contact stresses) $\sigma_{i k}$ and $\tau_{i k}$ are interpreted as the components of the specific force of mechanical response of the element $i$ to the mechanical loading by the neighboring element $k$. These stresses are the functions of the 
$i$-th element strains $\varepsilon_{i k}$ and $\gamma_{i k}$ in the pair $i-k$. We proposed the general form of these functions, which assumes homogeneous deformability of the element and linear relation between volume strain and mean stress (or pressure) [42-44]:

$$
\left\{\begin{array}{l}
\sigma_{i k}=\sigma_{i k}^{\text {pair }}\left(\varepsilon_{i k}, \dot{\varepsilon}_{i k}\right)+B_{i} \bar{\sigma}_{\text {mean }}^{i}=\sigma_{i k}^{\text {pair }}\left(\varepsilon_{i k}, \dot{\varepsilon}_{i k}\right)-B_{i} \bar{P}_{i} \\
\tau_{i k}=\tau_{i k}^{\text {pair }}\left(\gamma_{i k}, \dot{\gamma}_{i k}\right)
\end{array}\right.
$$

Here, the upper index "pair" denotes pair-wise function, $\bar{\sigma}_{\text {mean }}^{i}=-\bar{P}_{i}=$ $\left(\bar{\sigma}_{x x}^{i}+\bar{\sigma}_{y y}^{i}+\bar{\sigma}_{z z}^{i}\right) / 3, B_{i}$ is the material parameter. The first relation in (6) suggests that the normal (compressive/tensile) resistance of an element is determined by both the strain of this element $\varepsilon_{i k}$ along the loading axis and the hydrostatic (liquid-like) component. The second relation is written in the pair-wise approximation, which ideologically corresponds to the relations connecting the off-diagonal components of the stress and strain (or strain rate) tensors in most rheological models of solids. The specific form of the pair-wise components $\sigma_{i k}^{\text {pair }}$ and $\tau_{i k}^{\text {pair }}$ as well as the values of the material coefficients are determined by the applied rheological model of the material modeled by a discrete element. The necessity to satisfy Newton's third law $\left(\sigma_{i k}=\sigma_{k i}\right.$ and $\left.\tau_{i k}=\tau_{k i}\right)$ leads to the following systems of equations, which are used to calculate the current value of interaction forces $\vec{F}_{i k}^{c p}$ and $\vec{F}_{i k}^{t p}$ for the motion equation (1):

$$
\begin{gathered}
\left\{\begin{array}{l}
\sigma_{i k}^{\text {pair }}\left(\varepsilon_{i k}, \dot{\varepsilon}_{i k}\right)+B_{i} \bar{\sigma}_{\text {mean }}^{i}=\sigma_{k i}^{\text {pair }}\left(\varepsilon_{k i}, \dot{\varepsilon}_{k i}\right)+B_{i} \bar{\sigma}_{\text {mean }}^{i} \\
\Delta r_{i k}=\Delta q_{i k}+\Delta q_{k i}=R_{i} \Delta \varepsilon_{i k}+R_{k} \Delta \varepsilon_{k i}
\end{array}\right. \\
\left\{\begin{array}{l}
\tau_{i k}^{\text {pair }}\left(\gamma_{i k}, \dot{\gamma}_{i k}\right)=\tau_{k i}^{\text {pair }}\left(\gamma_{k i}, \dot{\gamma}_{k i}\right) \\
\Delta l_{i k}^{s h}=R_{i} \Delta \gamma_{i k}+R_{k} \Delta \gamma_{k i}
\end{array}\right.
\end{gathered}
$$

The solutions to each pair of equations are the values of pair strains. These strains are then used to calculate the current values of the forces of interaction of elements according to (6).

5. A pair of elements modeling a part of a consolidated material is assumed to be chemically bonded (linked). The central interaction of linked elements includes resistance to compression and tension, and the tangential interaction typically takes into account shear and bending resistance [42]. In the framework of the discrete element method, the elementary act of fracture at the considered spatial scale is the breaking of the chemical bond between the two elements. The condition of linked-to-unlinked transition is governed by a specified fracture criterion for a pair. This criterion is determined as a fracture condition at the contact area. Most fracture criteria in the mechanics of a deformable solid are formulated in force-like form in terms of the invariants of the stress tensor (Mises, Mohr-Coulomb, Drucker-Prager and other failure criteria). We proposed an approach to implementation of such kind of criteria within the framework of the formalism of deformable elements [42, 44]. It is based on determining the local stress tensor on the contact area of the linked pair of elements and 
calculating its invariants. The local stress tensor $\sigma_{\alpha^{\prime} \beta^{\prime}}^{i k}$ is determined in the local coordinate system of the pair $i-k$. The specific forces $\sigma_{i k}$ and $\tau_{i k}$ are used as the diagonal and off-diagonal components of this tensor. The missing 4 components are determined on the contact surface by linear interpolation of the corresponding components of average stress tensors in the interacting elements: $\sigma_{\alpha^{\prime} \beta^{\prime}}^{i k}=\left(\bar{\sigma}_{\alpha^{\prime} \beta^{\prime}}^{i} q_{k i}+\bar{\sigma}_{\alpha^{\prime} \beta^{\prime}}^{k} q_{i k}\right) / r_{i k}$. Here the accent means that average stresses are considered in the local coordinate system.

A bond break leads to a change in the interaction in a pair of discrete elements: the central interaction includes only compression resistance, and the dry [22] or viscous friction force is usually used as the tangential force. A pair becomes noninteracting if the value of the central force becomes equal to zero. We also note that the deformability of elements leads to a generalized formulation of contact detection condition, which takes into account a change in the linear dimensions of elements [42, 44].

6. Contact interaction of unlinked discrete elements is traditionally treated as nonadhesive. However, in many real systems, the adhesion of surfaces is an important factor determining the laws of friction and wear [45]. To adequately model the adhesive contact of surfaces, we assume attractive normal force acting between elements even after they are debonded [46, 47]. This force varies with separation of the surfaces of interacting elements according to prescribed model of adhesion (Dugdale's, Van-der-Vaals or other interaction potential). The value of separation is determined with taking into account deformation of elements along the normal $\vec{n}_{i k}$. The tangential force of interaction of unlinked and noncontact elements is assumed to be zero.

At large values of surface energy, the mechanical contact of chemically clean and smooth surfaces can be accompanied by the formation of a chemical bond (this effect is often called cold welding). This effect is taken into account in the method of deformable discrete elements based on the use of the criterion of unlinked-tolinked transition for contacting pairs of elements. The pair of elements becomes linked if the criterion is satisfied. We proposed some formulations of such kind of criteria including critical values of the contact normal stress and plastic work of deformation [42].

\section{Principles of Implementation of Rheological Models}

The most important advantage of the proposed general formulation of elementelement interaction (6)-(8) is the possibility of simple implementation of various rheological models of solids. One can see from relation (4) that the components of average stress tensor are linearly related to the forces of interaction of the element with its neighbors. In turn, the interaction forces are linearly related to the components of average stress tensor by relation (6). A fully similar interconnection takes place between average strains and pair strains of the element. We first showed that the similarity of the relationship between average and local (contact) stress and strain 
parameters of the element inevitably leads to the fact that the specific formulation of relations (6) should be similar to the formulation of the constitutive relations for the material of a discrete element. In other words, the relation for the specific central force $\sigma_{i k}$ of the $i$ th element response to the mechanical loading by the neighbor $k$ has to be formulated by means of direct rewriting of the constitutive equation for the diagonal components of the stress tensor. The specific force of tangential response $\tau_{i k}$ is formulated by direct rewriting the corresponding constitutive relation for off-diagonal stress components. Using these principles, we implemented macroscopic mechanical models of elasticity, viscoelasticity, and plasticity and applied them to study the behavior of various materials, including metals, ceramics, composite materials, rocks, rubbers, and even bone tissues [38, 42-44].

Below there is an example of the relations (6) for the quite general case of locally isotropic viscoelastic (described by the Prony series) material of the element. These relations are written in an incremental form:

$$
\left\{\begin{aligned}
\Delta \sigma_{i k}= & 2 G_{i, \Sigma} \Delta \varepsilon_{i k}+\left(1-\frac{2 G_{i}}{3 K_{i}}\right) \Delta \bar{\sigma}_{\text {mean }}^{i} \\
& -2 \sum_{p} \frac{G_{i, M p}}{\eta_{i, M p}} \Delta \sigma_{i k, M p}+2 \frac{\Delta \bar{\sigma}_{\text {mean }}^{i}}{3 K_{i, \Sigma}} \sum_{p} \frac{G_{i, M p} K_{i, M p}}{\eta_{i, M p}} . \\
\Delta \tau_{i k}= & 2 G_{i, \Sigma} \Delta \gamma_{i k}-2 \sum_{p} \frac{G_{i, M p}}{\eta_{i, M p}} \Delta \tau_{i k, M p}
\end{aligned}\right.
$$

Here $G_{i, \Sigma}=G_{i, K}+\sum_{p} G_{i, M}$ is the instant shear modulus of viscoelastic material $\left(G_{i, K}\right.$ is the shear modulus of the Kelvin element, $G_{i, M p}$ is the shear modulus of the $p$ th Maxwell element in a series), $K_{i, \Sigma}$ is the total bulk modulus determined in a similar way, $\eta_{i, M p}$ is the dynamic viscosity of the $p$-th Maxwell element, $\sigma_{i k, M p}$ and $\tau_{i k, M p}$ are the contributions of the $p$ th Maxwell element to the total specific force. It is easy to show that substitution of the relations (9) to the definition of average stress tensor (4) leads to rigorous fulfilment of the constitutive equation for the viscoelastic material in terms of average stresses and strains. The particular case of (9) is the linear-elastic model (the only Kelvin element), which is typically used to numerically study the elastic behavior of brittle and ductile materials.

It is well known that although the mechanisms of plasticity can qualitatively differ for the materials of various natures (defects of the crystal lattice in metals and alloys and discontinuities in microscopically brittle materials), their macroscopic inelastic behavior is adequately described on the basis of similar models based on the principles of the classical theory of plastic flow. In the framework of this theory, plasticity is described as instantaneous relaxation of "excess" stresses when the plasticity criterion (which is traditionally expressed in terms of the stress tensor invariants) exceeds a specified threshold value (yield shear stress).

Macroscopic inelastic (ductile) behavior of materials is conventionally modelled using associated (for metals and polymers) and non-associated (for ceramic materials, rocks and bone tissues under mechanical confinement) plastic flow models. 
The most popular way to implement these models within explicit numerical methods of continuum mechanics is the use of radial return algorithm of Wilkins [48]. We were the first to show that this algorithm can be easily adapted to the formalism of deformable discrete elements. By the analogy with elasticity, the prescribed law of mapping the average stresses $\bar{\sigma}_{\alpha \beta}^{i}$ is rigorously satisfied when applying the stress correcting expressions to the specific response forces $\sigma_{i k}$ and $\tau_{i k}$. Using this way, we numerically implemented the widely used macroscopic model of plasticity of metals with von Mises yield criterion [42, 43] and non-associated plastic flow model of Nikolaevsky [44] (the macroscopic rock plasticity model with von Mises-Schleicher yield criterion [49]). Other models of elasticity and plasticity can be implemented within the formalism of deformable discrete elements using this direct way. In particular, recently we developed a numerical model of inelastic deformation and fracture of brittle materials, which takes into account the finite incubation time of structural defects and applies principles of the structural-kinetic theory of strength [50].

The generality of the developed formalism allows one to implement not only mechanical but also coupled (thermomechanical, poromechanical and so on) material models. One of the most significant recent achievements of Professor S. G. Psakhie and his scientific team is the development of a hybrid (coupled) DEM-based technique to model the mechanical behavior of "contrast" materials, namely the porous materials with solid skeleton and interstitial liquid [38, 51]. Well-known examples of materials with locally contrasting mechanical properties are watered porous rocks and rubbers, bone and soft tissues. The importance of adequate consideration of the liquid phase in the contrast materials is determined by the fact that such kind of materials possesses strongly nonlinear behavior and non-stationary mechanical characteristics (even in the case of elastic-brittle skeleton) due to redistribution of mobile interstitial fluid in the pore space. Note that the formalism of DEM-based hybrid method was developed in collaboration with Dr. S. Zavsek (Velenje Coal Mine) and Professor J. Pezdic (University of Ljubljana).

Within the framework of this hybrid technique, the discrete element is considered as porous and permeable solid. The mechanical behavior of both solid skeleton and interstitial fluid and their mutual influence are taken into account. In particular, using Biot's linear model of poroelasticity [52] and Terzaghi's concept of effective stresses, we developed the coupled macroscopic model of permeable brittle materials. The main constitutive equations of this model are:

1. Dependence of the pore volume of an element on the hydrostatic component of the external load and the pore pressure

$$
\frac{\Omega_{p}-\Omega_{p 0}}{\Omega}=\phi-\phi_{0}=\frac{a}{K} \sigma_{m e a n}+\left(\frac{1}{K}-\frac{1+\phi}{K_{s}}\right) P_{\text {pore }},
$$

where $\Omega_{p}$ and $\Omega_{p 0}$ are the current and initial (in an undeformed element) values of the pore volume, $\varphi$ and $\varphi_{0}$ are the corresponding values of porosity, $\Omega$ is the volume of the element, $P_{\text {pore }}$ is the pore pressure in the volume of the element.

2. Constitutive equation of linear compressible liquid in the pore space 


$$
P_{\text {pore }}=P_{\text {pore }}^{0}+K_{f l}\left(\frac{\rho_{f l}}{\rho_{f l}^{o}}-1\right)=P_{\text {pore }}^{0}+K_{f l}\left(\frac{m_{f l}}{\rho_{f l}^{o} V_{p}}-1\right)
$$

where $\rho_{f l}^{0}$ and $P_{\text {pore }}^{0}$ are the equilibrium values of the density and pressure of the fluid under atmospheric conditions (in the absence of a mechanical confinement), $\rho_{f l}$ is the current value of the density of the liquid in the pore space of the element, $K_{f l}$ is the bulk modulus of the liquid.

3. Hooke's law for poroelastic material of the element

$$
\Delta \sigma_{\alpha \beta}=2 G\left(\Delta \varepsilon_{\alpha \beta}-\delta_{\alpha \beta} \frac{a \Delta P_{\text {pore }}}{K}\right)+\delta_{\alpha \beta}\left(1-\frac{2 G}{K}\right) \Delta \sigma_{\text {mean }},
$$

where $a=1-K / K_{s}$ is a coefficient of poroelasticity.

4. Modified formulations of yield and fracture criteria in terms of Terzaghi's effective stresses $\bar{\sigma}_{\alpha \beta}^{\text {eff }}=\bar{\sigma}_{\text {mean }}+P_{\text {pore }}[51]$.

5. The equations of motion of discrete elements (1) are supplemented by the classical equation of transport of interstitial fluid in the pore space of the material [53]. The transport equation is solved on an ensemble of discrete elements by the finite volume method [51].

Shown below examples demonstrate the capability of the developed formalism to implement various complex material models. This allows qualitative expansion of the range of simulated materials and the spatial scales under consideration.

\section{Recent Applications of the Formalism of Deformable Elements}

DEM is particularly efficient technique to study various aspects of the contact problems (including mechanisms of wear) in technical and natural friction pairs. One of the main principles of Professor Psakhie was a diversification of research activity and the use of the developed mathematical tools in a variety of scientific fields. The section is devoted to a brief outline of some recent results of computer study of contact problems in technical and biological systems. These studies were initiated and supervised by S. G. Psakhie in close collaboration with Professor V. L. Popov.

\subsection{Surface Adhesion as a Factor Controlling Regimes of Adhesive Wear}

Over the past two decades, the authors have carried out numerical studies of the laws of friction of rough surfaces of ductile and brittle materials. The key results of the 
studies are the features of the formation of the third body (quasi-liquid nano-layer) [54], the obtained generalized functional dependence of the friction coefficient on dimensionless combinations of material parameters and loading parameters $[54,55]$, the formulated principles of nanotribospectroscopy as a promising nondestructivetesting technique for assessment of nanostructured coating and surface layer damage [56].

Recent joint research in this field has focused on studying the "elementary" wear mechanisms (that is, the modes of fracture of individual asperities) and analyzing the effect of attractive (adhesive) force between spatially separated surfaces on the involved mechanism of asperity wear under the condition of low-angle collision. Note that adhesive wear of the surface layers of contacting bodies is a widely studied but still poorly predicted phenomenon [45]. A key factor determining the regime and the rate of wear is the adhesive interaction of surfaces in the contact spots of asperities. Here, the term "adhesive interaction" includes (a) attractive interaction between detached surfaces and (b) effect of "cold welding" (chemical bonding of contacting surfaces) for chemically clear surfaces with high surface energy. The first well-known attempt to generalize the patterns of adhesive wear is the analytical model of Rabinowicz [57, 58]. He examined two qualitatively different mechanisms of asperity wear (plastic smoothing and breakaway) and showed that the involving of a specific mechanism is determined by the size parameter, which is a combination of specific material parameters, including shear strength, elastic constants, and specific surface energy. In recent years, a number of scientific groups carried out extensive numerical studies of the laws of interaction of single asperities [59,60]. These studies have shown that the dependence of the power of asperity wear on material parameters generally has a significantly more complex nonlinear form, and the spectrum of realized mechanisms is not limited to those considered by Rabinowicz. However, the vast majority of theoretical results were obtained for nanoscale asperities using atomistic simulation.

We were the first to make a systematic numerical analysis of typical modes of asperity wear on higher (micro- and mesoscopic) scales. Due to the capabilities of the developed formalism of deformable discrete elements, such a study was carried out for ductile and brittle materials.

The main result of the study is that we revealed and substantiated two dimensionless material parameters that control the regime of asperity wear [46].

The first one is the ratio of attractive stress $\sigma_{0}$ between the detached surfaces (the adhesion stress) to the shear strength of the material $\sigma_{j}$. We showed that the range of values of this dimensionless parameter could be divided into two intervals with its own wear regime in each of them. The border value of the ratio $\left(\sigma_{0}^{*} / \sigma_{j}\right)$ and the specific involved mechanisms (specific modes) of asperity wear are determined by the value of the second dimensionless parameter.

The second one is the dimensionless parameter $a$, which characterizes shear strength sensitivity to the applied normal stress (or mean stress). It can be expressed in terms of the ratio of material strength values under different loading conditions. Note that shear strength sensitivity parameter effectively characterizes fracture toughness and is closely related to material brittleness: $a \approx 1$ for highly ductile materials, 
$1<a<1.5$ for moderately ductile materials, $a$ reaches 5-10 for elastic-brittle solids. We showed that increase in the ratio of adhesion stress to shear strength is accompanied by the transitions:

1. from slipping (wear at the atomic scale) to grinding-based wear regime for the case of highly and moderately ductile materials;

2. from breakaway (separation of the asperity from the foundation) to grindingbased wear regime for the case of materials with a limited ductility or brittle.

We have built the qualitative map of asperity wear regimes in terms of dimensionless material parameters $\sigma_{0} / \sigma_{j}$ and $a$ [46]. Figure 3 shows a rough schematic representation of this classification of wear regimes, which agrees with the results of atomistic studies by other researchers, supplements and generalizes them to higher scales of surface roughness.

An important result of the DEM-based study is the determined dependence of the position of the boundary between the wear regimes (the border value $\sigma_{0}^{*} / \sigma_{j}$ ) separating "low adhesion" and "high adhesion" wear modes) on asperity size $L$. We showed that the value is determined not by the absolute value of $L$, but its relation to the length $d$ of action of attractive potential between spatially separated surfaces [47]. This dependence has a nonlinear increasing profile with reaching a saturation level at the scale $\sim 10^{4}$ of the ratio $L / d$. For larger asperities $\left(L / d>10^{4}\right)$, the results of the analysis of the wear regimes are scale invariant under the condition of scale invariance of the mechanical characteristics of the material.

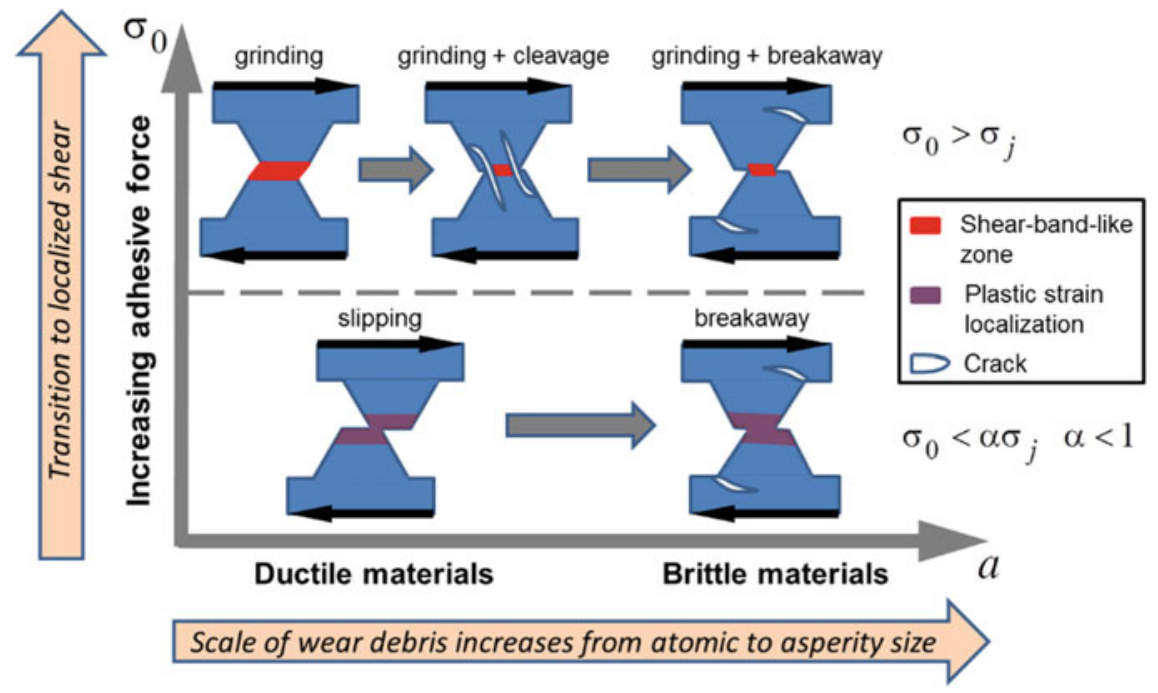

Fig. 3 Schematic classification of wear regimes of asperities for the case of tangential dry contact. The coefficient $\alpha$ is close to 1 for highly ductile materials and about $0.5-0.6$ for brittle materials $\left(\alpha=\sigma_{0}^{*} / \sigma j\right)[46]$. 
So, the surface adhesion stress is a criterion that determines the wear regime of asperities under the condition of tangential contact. The obtained map of asperity wear (Fig. 3) together with the revealed asperity size (scale) effect have both fundamental and practical significance as they allow forecasting the dominating mode of asperity wear for quite different materials from brittle to highly ductile.

\subsection{Influence of Interstitial Fluid on the Sensitivity of the Femur to the Rate of Contact Loading}

Contact loading is capable of determining not only surface wear and structural modification of surface layers, but also structural changes in the volume of contacting bodies. This is particularly relevant for biological (bone and cartilage) tissues. Functioning of these tissues is largely determined by the redistribution of interstitial fluid. Pore fluid has a complex nonlinear effect on the state and behavior of these biological materials. There are two key aspects of this influence. The first one is fluid flow in the pore space. Fluid flow provides the transfer of nutrients and oxygen and serves as a prerequisite for cell proliferation and tissue regeneration [61, 62]. The second aspect is the mechanical effect of pore pressure. It causes local tensile stresses in the skeleton and contributes to local fracture and gradual degradation of bone tissue. At extreme values of pore pressure it makes a significant contribution to the formation of cracks. The aforesaid argues the existence of optimal distributions of pore fluid (that is, optimal maximum local values and their gradient), which on the one hand provide sufficient fluid flows to ensure normal (regenerative) tissue activity, and on the other hand do not cause local fracture. Such distribution is formed under certain ("optimal") condition of the mechanical (contact) loading of the analyzed organ.

This problem is especially important in application to elements of the human musculoskeletal system (joints) because functioning of bone tissues in these regions strongly depends on the normal contact load and mode of tangential contact interaction. One of the topical problems in this field is the analysis of stress evolution and redistribution of interstitial fluid in the femur near the hip joint under dynamic loading. The developed formalism of permeable fluid-saturated discrete elements is an efficient tool for such a dynamic analysis up to the stage of macroscopic crack formation. Note that despite the topicality of the problem, there are practically no works devoted to the numerical study of the dynamic mechanical response of femur within the consideration of bone tissue as a multiphase fluid-saturated solid. The preliminary results of the numerical DEM-based study presented below are, therefore, pioneering in some way.

The aim of the study is to determine the loading rate sensitivity of healthy bone tissue of the proximal femur and bone tissue at different stages of osteoporosis. The main attention was paid to the regions of the femur in which the volume stresses are positive. This is due to the fact that bone is a brittle material and is characterized by 


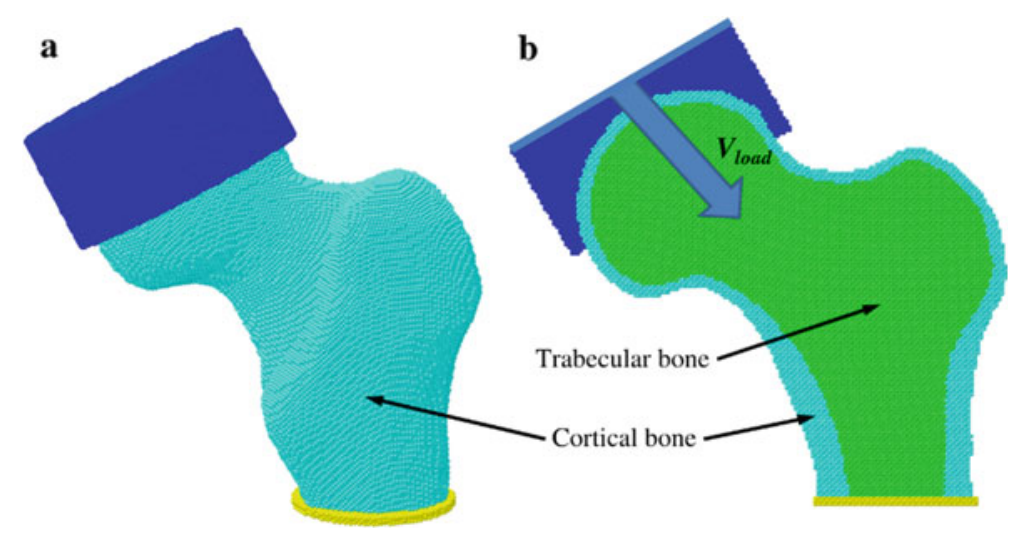

Fig. 4 General view of the model of the proximal femur (a) and its cross section (b) with differently colored cortical and spongy (trabecular) bone tissues

significantly lower tensile strength than compressive one. First of all, we analyzed the femoral neck (the typical region of the origin of the cracks).

The femur was modeled as a heterogeneous (shell) structure containing two fluidsaturated porous layers (Fig. 4): an inner spongy bone with low stiffness and an outer layer of cortical bone tissue. The latter plays the role of a hard shell and determines constrained conditions for the deformation of the soft spongy bone. The standard CAD model of the femur was used as the geometric basis of the DEM model [63]. We modelled compression of the proximal part of the femur along its main physiological direction (at an angle of about $45^{\circ}$ relative to the orientation of the stem, Fig. 4b). The load was applied by setting the constant velocity $V_{\text {load }}$ to the outermost elements of a special "cap" on the bone head. The base of the model was fixed. The model was loaded until the resistance force $F_{\max }=10 \mathrm{kN}$ was reached $(10 \mathrm{kN}$, is about $70 \%$ of the critical load [64]). A velocity range from 1 to $8 \mathrm{~m} / \mathrm{s}$ was considered. This range covers various modes of motor activity up to the "extreme" one.

Various authors previously showed that the strain rate dependence of the mechanical characteristics of heterogeneous (block-structured) brittle materials under confined loading conditions has not just a non-linear, but non-monotonic profile with a local minimum [65]. The reason for the non-monotonic nature of the dependence is the competition of two factors: pore fluid flow, which affects the redistribution of local stresses in the skeleton, and change in the equilibrium linear dimensions of the material due to change in pore pressure. The non-monotonic nature of the influence of interstitial fluid is especially pronounced under conditions of constrained deformation, when a change in linear dimensions causes a corresponding change in the degree of constraint. The biomechanical system under consideration (femur) also has a heterogeneous structure and is constrained by a hard cortical bone "shell". The shell causes confined deformation of a much softer inner region. This gives reason to suggest that under dynamic loading of such a system, the dependence of local stress values (including stresses in the femoral neck) on the strain rate can also 
be nonmonotonic with a local minimum. The magnitude of corresponding "optimal" loading velocity should be related to the characteristics of the porosity and permeability of the bone.

The simulation results confirmed these assumptions and, moreover, allowed us to estimate the characteristic values of the "optimal" loading velocity for healthy bone and bone at various stages of osteoporosis. The key result of the numerical study is the revealed non-linear and nonmonotonic dependence of the stress concentration in the femoral neck on the loading rate. Figure 5 shows examples of the distribution of mean and equivalent stresses in a healthy femur sample at different loading velocities. The simulation results show that an increase in $V_{\text {load }}$ is accompanied by an increase in the values of the parameters characterizing the stress concentration in the upper part of the femoral neck. In particular, the volume of the region of maximum stresses and the magnitude of the maximum stress in the neck increase. However, when approaching the "optimal" value of the loading velocity $(\sim 3 \mathrm{~m} / \mathrm{s}$ for the healthy bone), stress concentration decreases, then reaches minimum value at the "optimal" velocity, and then (with further increase in $V_{\text {load }}$ ) increases monotonously again. This effect takes place both for mean and equivalent stresses. The relative magnitude of the reduction in peak stresses in the neck reaches 10-20\%, and the maximum decrease in the volume of the region of stress concentration amounts to $10 \%$. Note that the loading velocities 3-4 m/s correspond to the regime of training motion of a human.

The described effect of reducing the heterogeneity of the stress state of bone tissue (particularly in the femoral neck) in the vicinity of the "optimal" loading velocity is directly related to the influence of pore fluid pressure and redistribution. Special simulations for the "dry" femur showed a monotonic dependence of the stress

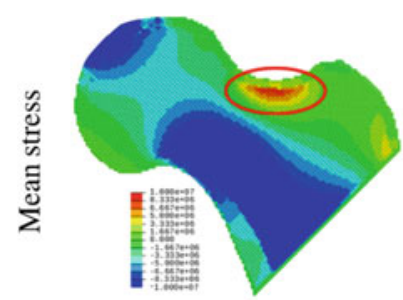

a

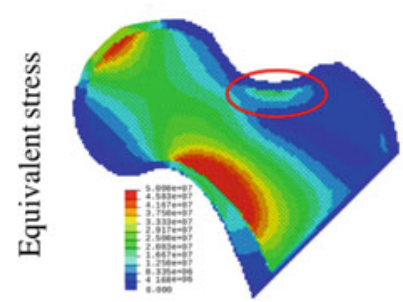

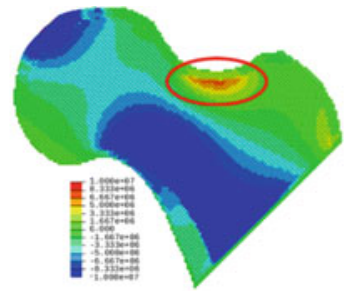

b



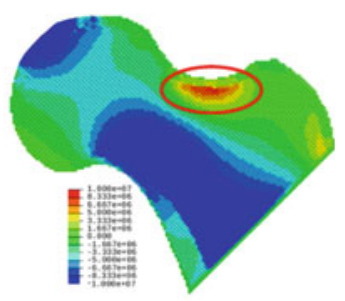

c

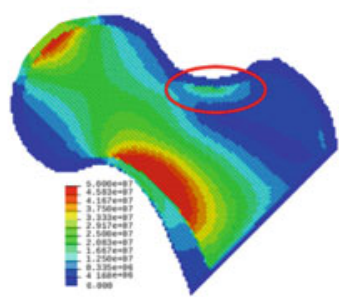

Fig. 5 Distributions of mean stress (upper row) and equivalent stress (lower row) in the proximal femur at different values of loading velocity: a $1 \mathrm{~m} / \mathrm{s} ; \mathbf{b} 3 \mathrm{~m} / \mathrm{s} ; \mathbf{c} 5 \mathrm{~m} / \mathrm{s}$. All pictures correspond to the same value of applied load $F_{\max }=10 \mathrm{kN}$ 
concentration in the sample (including the femoral neck) on the loading rate in the entire considered velocity range.

Preliminary numerical studies have also shown that a change in bone tissue parameters corresponding to the successive stages of osteoporosis leads to a systematic increase in the values of "optimal" loading velocity. Bone tissue suffering from osteoporosis is characterized by the reduced strength and higher brittleness. Therefore, our results indicate that the selection of the optimal regime of motor activity for such a femur is questionable.

Finally, we note the importance of the revealed effect of the "optimal" loading rate. Despite the relatively small decrease in the maximum tensile stresses in the critical region of the bone (upper part of the neck), this effect can have a significant positive value in the long term (with multiple repetitions of the load). In addition to reducing the risk of microdamage nucleation/accumulation in the upper part of the neck, a decrease in the magnitude of positive volume stresses contributes to a more intensive and uniform circulation of interstitial fluid in this region of the bone. The consequence is a more complete and rational flow of substances necessary for the normal functioning of bone tissue at optimal loading intensity. So, the results obtained are critically important for determining the optimal modes of motor activity of people, as well as for developing strategies for treating osteoarthritis and preventing the negative consequences of osteoporosis.

\section{Conclusion}

A formalism of homogeneously deformable elements, which was developed by Professor Sergey G. Psakhie and his colleagues, made possible qualitative enhancement of the capabilities of the particle-based discrete element method. The main advantage of DEM is the ability to correctly describe the mechanical behavior of various materials with taking into account the accompanying thermal, hydromechanical and other effects. Deformability of elements is of particular importance when studying various aspects of dynamic contact interaction, for example, stick-toslip transition in technical and geological contact zones, mechanisms of friction and wear, redistribution of pore fluid in the surface layers of geological and biological joints, etc.

Two key factors that identified the advantages of the formalism of deformable elements should be especially noted. The first one is the postulation of the manyparticle form of relations for the element-element interaction forces. In this regard, DEM has much in common with molecular dynamics method. Pair-wise interatomic potentials are able to catch basic properties of crystal lattice, but do not describe many fundamentally important effects in the bulk and on the surface of solids, including those determining plasticity and phase transformations. Note that the approximation of a homogeneously deformable element is an efficient alternative to more computationally expensive combined DEM-FEM technique. The second factor is the proposed universal method for determining the specific form (and the values of 
constants) of the interaction forces. This method is based on the replication of the corresponding rheological relationships of the applied mechanical model. It "circumvents" the fundamental problem of traditional DEM, namely, the need to find a vector analogue of constitutive equations written in tensor form. The particular cases of rheological models mentioned in this chapter are related to locally isotropic materials, however, models of elasticity, ductility and local fracture of anisotropic materials can be implemented in a similar way.

At present time, the formalism of deformable DEM is actively developed and applied in various fields and in the wide range of spatial scales. Moreover, international scientific teams leaded by well-recognized scholars adopt these ideas and develop them in their own way. DEM with deformable elements becomes not just an addition to the classical continuum numerical methods, but to some extent their competitive even in the areas of mechanics, where they traditionally dominate.

Acknowledgements The work was performed according to the Government research assignment for ISPMS SB RAS.

\section{References}

1. Cauchy AL (1823) Recherches sur l'equilibre et le mouvement interieur des corps solides ou fluides, elastiques ou non lastiques. Bulletin des sciences par la Societe Philomatique de Paris 9-13

2. Navier CL (1823) Sur les lois de l'equilibre et du mouvement des corps solides elastiques. Bulletin des sciences par la Societe Philomatique de Paris 177-181

3. Kocsis A, Challamel N (2018) Generalized models and non-classic approaches in complex materials 1. Springer, Berlin, pp 451-486 (On the foundation of a generalized nonlocal extensible shear beam model from discrete interactions)

4. Zienkiewicz OC, Taylor RL, Fox DD (2014) The finite element method for solid and structural mechanics, 7th edn. Butterworth-Heinemann, Oxford

5. Balokhonov RR, Romanova VA, Kulkov AS (2020) Microstructure-based analysis of deformation and fracture in metal-matrix composite materials. Eng Fail Anal 110:104412. https:// doi.org/10.1016/j.engfailanal.2020.104412

6. Moczo P, Kristek J, Galis M (2014) The finite-difference modelling of earthquake motions: waves and ruptures. Cambridge University Press, Cambridge (UK)

7. Garavand A, Stefanov YP, Rebetsky YL, Bakeev RA, Myasnikov AV (2020) Numerical modeling of plastic deformation and failure around a wellbore in compaction and dilation modes. Int J Numer Anal Meth Geomech 44(6):1-28. https://doi.org/10.1002/nag.3041

8. Cheng AHD, Syngellakis S (eds) (2019) Boundary elements and other mesh reduction methods XLI. WIT Press, Southampton (UK)

9. Popov VL, Pohrt R, Li Q (2017) Strength of adhesive contacts: influence of contact geometry and material gradients. Friction 5(3):308-325. https://doi.org/10.1007/s40544-017-0177-3

10. Yazid A, Abdelkader N, Abdelmajid H (2009) A state-of-the-art review of the X-FEM for computationalfracture mechanics. Appl Math Model 33(12):4269-4282. https://doi.org/10. 1016/j.apm.2009.02.010

11. Pohrt R, Popov VL (2015) Adhesive contact simulation of elastic solids using local meshdependent detachment criterion in boundary elements method. Facta Univ Mech Eng 13(1):310 
12. Liu MB, Liu GR (2010) Smoothed particle hydrodynamics (SPH): an overview and recent developments. Arch Comput Methods Eng 17:25-76. https://doi.org/10.1007/s11831-0109040-7

13. Li X, Zhao J (2019) An overview of particle-based numerical manifold method and its application to dynamic rock fracturing. J Rock Mech Geotech Eng 11(3):684-700. https://doi.org/ 10.1016/j.jrmge.2019.02.003

14. Nabian MA, Farhadi L (2016) Multiphase mesh-free particle method for simulating granular flows and sediment transport. J Hydraul Eng 143(4):04016102. https://doi.org/10.1061/(ASC E)HY.1943-7900.0001275

15. Munjiza A, Smoljanović H, Živaljić N, Mihanovic A, Divić V, Uzelac I, Nikolić Ž, Balić I, Trogrlić B (2019) Structural applications of the combined finite-discrete element method. Comput Part Mech. https://doi.org/10.1007/s40571-019-00286-5

16. Rodriguez JM, Carbonell JM, Cante JC, Oliver J (2016) The particle finite element method (PFEM) in thermo-mechanical problems. Int J Numer Meth Eng 107(9):733-785. https://doi. org/10.1002/nme.5186

17. Cerquaglia ML, Deliege G, Boman R, Papeleux L, Ponthot JP (2017) The particle finite element method for the numerical simulation of bird strike. Int J Impact Eng 109:1-13. https://doi.org/ 10.1016/j.ijimpeng.2017.05.014

18. Jing L, Stephansson O (2007) Fundamentals of discrete element method for rock engineering: theory and applications. Elsevier, Amsterdam (NL)

19. Bicanic N (2017) Encyclopaedia of computational mechanics, 2nd edn. Wiley, Hoboken, pp 1-38 (Discrete element methods)

20. Cundall PA, Strack ODL (1979) A discrete numerical model for granular assemblies. Geotechnique 29(1):47-65

21. Cundall PA, Hart RD (1992) Numerical modelling of discontinua. Eng Comput 9(2):101-113. https://doi.org/10.1108/eb023851

22. Potyondy DO, Cundall PA (2004) A bonded-particle model for rock. Int J Rock Mech Min Sci 41:1329-1364. https://doi.org/10.1016/j.ijrmms.2004.09.011

23. Ivars DM, Pierce ME, Darcel C, Reyes-Montes J, Potyondy DO, Young RP, Cundall PA (2011) The synthetic rock mass approach for jointed rock mass modelling. Int J Rock Mech Min Sci 48(2):219-244. https://doi.org/10.1016/j.ijrmms.2010.11.014

24. Mustoe GGW (1992) A generalized formulation of the discrete element method. Eng Comput 9(2):181-190. https://doi.org/10.1108/eb023857

25. Hatzor YH, Ma G, Shi G-H (2017) Discontinuous deformation analysis in rock mechanical practice. CRC Press, Boca Raton (Florida, US)

26. Schneider B, Ramm E (2019) Conceptual experiments and discrete element simulations with polygonal particles. Granular Matter 21(91). https://doi.org/10.1007/s10035-019-0930-6

27. Podlozhnyuk A, Pirker S, Kloss C (2017) Efficient implementation of superquadric particles in discrete element method within an open-source framework. Comput Part Mech 4:101-118. https://doi.org/10.1007/s40571-016-0131-6

28. Sinaie S (2017) Application of the discrete element method for the simulation of size effects in concrete samples. Int J Solids Struct 108:244-253. https://doi.org/10.1016/j.ijsolstr.2016. 12.022

29. Potyondy DO (2015) The bonded-particle model as a tool for rock mechanics research and application: current trends and future directions. Geosyst Eng 18(1):1-28. https://doi.org/10. 1080/12269328.2014.998346

30. Nosewicz S, Rojek J, Pietrzak K, Chmielewski M (2013) Viscoelastic discrete element model of powder sintering. Powder Technol 246:157-168. https://doi.org/10.1016/j.powtec.2013.05.020

31. Rojek J, Lumelskyj D, Nosewicz S, Romelczyk-Baishya B (2019) Numerical and experimental investigation of an elastoplastic contact model for spherical discrete elements. Comput Part Mech 6:383-392. https://doi.org/10.1007/s40571-018-00219-8

32. Wang G, Al-Ostaz A, Cheng AH-D, Mantena PR (2009) Hybrid lattice particle modeling: theoretical considerations for a $2 \mathrm{D}$ elastic spring network for dynamic fracture simulations. Comput Mater Sci 44(4):1126-1134. https://doi.org/10.1016/j.commatsci.2008.07.032 
33. Puglia VB, Kosteski LE, Riera JD, Iturrioz I (2019) Random field generation of the material properties in the lattice discrete element method. J Strain Anal Eng Des 54(4):236-246. https:// doi.org/10.1177/0309324719858849

34. Pieczywek PM, Zdunek A (2017) Compression simulations of plant tissue in 3D using a massspring system approach and discrete element method. Soft Matter 13:7318-7331. https://doi. org/10.1039/C7SM01137G

35. Zabulionis D, Rimsa V (2018) A lattice model for elastic particulate composites. Materials 11(9):1584. https://doi.org/10.3390/ma11091584

36. Rizvi ZH (2019) Lattice element method and its application to multiphysics. Dissertation in fulfilment of the requirements for the degree "Dr.-Ing." of the Faculty of Mathematics and Natural Sciences at Kiel University, Christian-Albrechts-Universität, Kiel

37. Psakhie SG, Horie Y, Ostermeyer GP, Korostelev SYu, Smolin AYu, Shilko EV, Dmitriev AI, Blatnik S, Špegel M, Zavšek S (2001) Movable cellular automata method for simulating materials with mesostructured. Theoret Appl Fract Mech 37(1-3):311-334

38. Psakhie SG, Shilko EV, Smolin AY, Dimaki AV, Dmitriev AI, Konovalenko IS, Astafurov SV, Zavshek S (2011) Approach to simulation of deformation and fracture hierarchically organized heterogeneous media, including contrast media. Phys Mesomech 14(1-5):224-248. https://doi. org/10.1016/j.physme.2011.12.003

39. Psakhie SG, Horie Y, Korostelev SYu, Smolin AYu, Dmitriev AI, Shilko EV, Alekseev SV (1995) Method of movable cellular automata as a tool for simulation within the framework of mesomechanics. Russ Phys J 38(11):1157-1168. https://doi.org/10.1007/BF00559396

40. Psakhie SG, Shilko EV, Smolin AY, Dmitriev AI, Korostelev SY (1996) Computer aided study of reaction-assisted powder mixture shock compaction at meso-scale. New computational technique. In: Proceedings of US-Russian workshop "Shock induced chemical processing", Saint-Petersburg, 23-24 June, 1996

41. Popov VL, Psakhie SG (2001) Theoretical principles of modeling elastoplastic media by movable cellular automata method. I. Homogeneous media. Phys Mesomech 4(1):15-25

42. Psakhie S, Shilko E, Smolin A, Astafurov S, Ovcharenko V (2013) Development of a formalism of movable cellular automaton method for numerical modeling of fracture of heterogeneous elastic-plastic materials. Frattura Ed Integrità Strutturale 24(7):26-59. https://doi.org/10.3221/ IGF-ESIS.24.04

43. Shilko EV, Psakhie SG, Schmauder S, Popov VL, Astafurov SV, Smolin AYu (2015) Overcoming the limitations of distinct element method for multiscale modeling of materials with multimodal internal structure. Comput Mater Sci 102:267-285. https://doi.org/10.1016/j.com matsci.2015.02.026

44. Psakhie SG, Shilko EV, Grigoriev AS, Astafurov SV, Dimaki AV, Smolin AYu (2014) A mathematical model of particle-particle interaction for discrete element based modeling of deformation and fracture of heterogeneous elastic-plastic materials. Eng Fract Mech 130:96-115. https://doi.org/10.1016/j.engfracmech.2014.04.034

45. Wen S, Huang P (2017) Principles of tribology, 2nd edn. Wiley, London (UK)

46. Dimaki AV, Shilko EV, Dudkin IV, Psakhie SG, Popov VL (2020) Role of adhesion stress in controlling transition between plastic, grinding and breakaway regimes of adhesive wear. Sci Rep 10:1585. https://doi.org/10.1038/s41598-020-57429-5

47. Dimaki AV, Dudkin IV, Popov VL, Shilko EV (2019) Influence of adhesion force and strain hardening coefficient of the material on the rate of adhesive wear in a dry tangential frictional contact. Russ Phys J 62(8):1398-1408. https://doi.org/10.1007/s11182-019-01857-y

48. Wilkins ML (1999) Computer simulation of dynamic phenomena. Springer, Berlin

49. Nikolaevsky VN (1996) Geomechanics and fluidodynamics with application to reservoir engineering. Kluwer Academic Publishers (Springer), Berlin

50. Grigoriev AS, Shilko EV, Skripnyak VA, Psakhie SG (2019) Kinetic approach to the development of computational dynamic models for brittle solids. Int J Impact Eng 123:14-25. https:// doi.org/10.1016/j.ijimpeng.2018.09.018

51. Psakhie SG, Dimaki AV, Shilko EV, Astafurov SV (2016) A coupled discrete element-finite difference approach for modeling mechanical response of fluid-saturated porous materials. Int J Numer Meth Eng 106(8):623-643. https://doi.org/10.1002/nme.5134 
52. Detournay E, Cheng AH-D (1993) Comprehensive rock engineering: principles, practice and projects. Pergamon Press, Oxford (UK), pp 113-171 (Fundamentals of poroelasticity)

53. Basniev KS, Dmitriev NM, Dmitriev NM, Chilingar GV (2012) Mechanics of fluid flow. Wiley, London (UK)

54. Popov VL, Psakhie SG, Dmitriev AI, Shilko E (2003) Quasi-fluid nano-layers at the interface between rubbing bodies: simulations by movable cellular automata. Wear 254(9):901-906. https://doi.org/10.1016/S0043-1648(03)00244-8

55. Bucher F, Dmitriev AI, Ertz M, Knothe K, Popov VL, Psakhie SG, Shilko EV (2006) Multiscale simulation of dry friction in wheel/rail contact. Wear 261(7-8):874-884. https://doi.org/10. 1016/j.wear.2006.01.046

56. Psakhie SG, Shilko EV, Popov VL, Starcevic J, Thaten J, Astafurov SV, Dimaki AV (2009) Assessment of nanostructured ceramic coating damage Nanotribospectroscopy. Russ Phys J 52(4):380-385. https://doi.org/10.1007/s11182-009-9242-3

57. Rabinowicz E (1958) The effect of size on the looseness of wear fragments. Wear 2(1):4-8

58. Popova E, Popov VL, Kim DE (2018) 60 years of Rabinowicz' criterion for adhesive wear. Friction 6(3):341-348. https://doi.org/10.1007/s40544-018-0240-8

59. von Lautz J, Pastewka L, Gumbsch P, Moseler M (2016) Molecular dynamic simulation of collision-induced third-body formation in hydrogen-free diamond-like carbon asperities. Tribol Lett 63:26. https://doi.org/10.1007/s11249-016-0712-9

60. Molinari J-F, Aghababaei R, Brink T, Frérot L, Milanese E (2018) Adhesive wear mechanisms uncovered by atomistic simulations. Friction 6:245-259. https://doi.org/10.1007/s40544-0180234-6

61. Christen P, Ito K, Ellouz R, Boutroy S, Sornay-Rendu E, Chapurlat RD, van Rietbergen B (2014) Bone remodelling in humans is load-driven but not lazy. Nat Commun 5:4855. https:// doi.org/10.1038/ncomms5855

62. Wittkowske C, Reilly GC, Lacroix D, Perrault CM (2016) In vitro bone cell models: impact of fluid shear stress on bone formation. Front Bioeng Biotechnol 4:87. https://doi.org/10.3389/ fbioe.2016.00087

63. Cheung G, Zalzal P, Bhandari M, Spelt JK, Papini M(2004) Finite element analysis of a femoral retrograde intramedullary nail subject to gait loading. Med Eng Phys 26(2):93-108. https:// doi.org/10.1016/j.medengphy.2003.10.006

64. Todo M (2018) Biomechanical analysis of hip joint arthroplasties using CT-image based finite element method. J Surg Res 1:34-41

65. Shilko EV, Dimaki AV, Psakhie SG (2018) Strength of shear bands in fluid-saturated rocks: a nonlinear effect of competition between dilation and fluid flow. Sci Rep 8:1428. https://doi. org/10.1038/s41598-018-19843-8

Open Access This chapter is licensed under the terms of the Creative Commons Attribution 4.0 International License (http://creativecommons.org/licenses/by/4.0/), which permits use, sharing, adaptation, distribution and reproduction in any medium or format, as long as you give appropriate credit to the original author(s) and the source, provide a link to the Creative Commons license and indicate if changes were made.

The images or other third party material in this chapter are included in the chapter's Creative Commons license, unless indicated otherwise in a credit line to the material. If material is not included in the chapter's Creative Commons license and your intended use is not permitted by statutory regulation or exceeds the permitted use, you will need to obtain permission directly from the copyright holder.

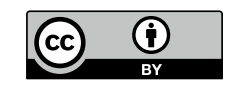

